\title{
GEOMETRIC AND DIFFERENTIAL PROPERTIES OF SUBANALYTIC SETS
}

\author{
EDWARD BIERSTONE AND PIERRE D. MILMAN
}

\begin{abstract}
We announce solutions of two fundamental problems in differential analysis and real analytic geometry, on composite differentiable functions and on semicoherence of subanalytic sets. Our main theorem asserts that the problems are equivalent and gives several natural necessary and sufficient conditions in terms of semicontinuity of discrete local invariants and metric properties of a closed subanalytic set.
\end{abstract}

\section{INTRODUCTION}

The results announced here include solutions of two fundamental problems in differential analysis and real analytic geometry, on $\mathscr{C}^{\infty}$ functions composed with a proper real analytic mapping, and on formal semicoherence of subanalytic sets (a stratified real version of the coherence theory of Oka and Cartan).

The composite function problem. Let $\varphi: M \rightarrow N$ be a proper (or semiproper) real analytic mapping, and let $\varphi^{*}: \mathscr{C}^{\infty}(N) \rightarrow$ $\mathscr{C}^{\infty}(M)$ denote the homomorphism of rings of $\mathscr{C}^{\infty}$ functions given by composition with $\varphi$. Is $\varphi^{*} \mathscr{C}^{\infty}(N)$ closed in $\mathscr{C}^{\infty}(M)$ (where the spaces have the $\mathscr{C}^{\infty}$ topology)? This problem was formulated by Thom and Glaeser [10]. It depends only on the image of $\varphi$, which is a closed subanalytic set $[2,3.5]$.

Subanalytic sets are the real analytic analogues of complex analytic and real semialgebraic sets, and share many of their important properties. (See, for example, [6].) But real algebraic sets already do not enjoy the coherence properties of complex analytic sets. And subanalytic sets, in general, differ in a crucial way from semialgebraic (or semianalytic) sets: The local topological dimension

Received by the editors December 20, 1990 and, in revised form, April 20, 1991.

1980 Mathematics Subject Classification (1985 Revision). Primary 32B20, 58C27; Secondary 32C42, 32K15.

Research partially supported by NSERC operating grants OGP0009070 and A8849. 
of a subanalytic set and the dimensions of its local analytic and formal closures may all be distinct [7]. Hironaka [11] announced that every subanalytic set satisfies a formal semicoherence property (weaker than that considered here or in $[5, \S 6]$ ) but Pawlucki has given a counterexample to Hironaka's assertion [15].

Let $\mathscr{F}_{b}(X)$ denote the ideal in $\hat{\mathscr{O}}_{b}$ of the formal closure of a subanalytic subset $X$ of $\mathbb{R}^{n}$, at a point $b \in \mathbb{R}^{n}$. (See Lemma 1 below. $\hat{\mathscr{O}}_{b}$ is the ring of formal power series at $b$.) If $X$ is semianalytic, then $\mathscr{F}_{b}(X)$ is generated by the ideal $\mathscr{A}_{b}(X)$ of germs of analytic functions that vanish on $X$ near $b$. Local properties of $X$ are studied classically in terms of the variation of these ideals with respect to $b$. (For example, coherence of complex analytic $X$, or Zariski semicontinuity of local invariants like the Hilbert-Samuel function of $\hat{\mathscr{O}}_{b} / \mathscr{F}_{b}(X)$, for complex or coherent real analytic $X$.) An important local metric characteristic of $X$ is the relationship between the order of vanishing of a germ of an analytic function $f$ at $b$ when restricted to $X$, and its order of vanishing modulo the formal local ideal; i.e., the relationship between the numbers

$$
\begin{aligned}
& \mu_{X, b}(f)=\sup \left\{p \in \mathbb{R}:|f(x)| \leq \text { const }|x-b|^{p}, x \in X\right\}, \\
& \nu_{X, b}(f)=\max \left\{p \in \mathbb{N}: f \in \hat{\mathfrak{m}}_{b}^{p}+\mathscr{F}_{b}(X)\right\},
\end{aligned}
$$

where $\hat{\mathfrak{m}}_{b}$ denotes the maximal ideal of $\hat{\mathscr{O}}_{b}$ (cf. [12]).

Our main result (Theorem 3 below) not only asserts that the composite function and formal semicoherence properties of a closed subanalytic set $X$ are equivalent, but also gives several natural necessary and sufficient conditions for these properties in terms of the variation of the ideals $\mathscr{F}_{b}(X)$; for example, semicontinuity (in a subanalytic sense) of the Hilbert-Samuel function of $\hat{\mathscr{O}}_{b} / \mathscr{F}_{b}(X)$, and bounds that are uniform with respect to $b$ on $\mu_{X, b}(f)$ in terms of $\nu_{X, b}(f)$. By Pawlucki's counterexample to semicoherence, none of the properties of Theorem 3 holds in general. (Pawłucki has shown directly that the composite function property fails for his example.) Closed subanalytic sets satisfying the composite function property have an important linear extension property (Corollary 4).

The class of closed Nash subanalytic sets (see $\S 3$ ) is the largest class previously known to satisfy the conditions of Theorem 3; see [2-5] for proofs and for complete histories of the problems treated here. Theorem 3 completes the program begun in these articles. Every semianalytic set is Nash. If $X$ is Nash, then for 
each point $b$, there are $\alpha_{b} \geq 1$ and $\beta_{b} \geq 0$ such that $\mu_{X, b}(\cdot) \leq$ $\alpha_{b} \nu_{X, b}(\cdot)+\beta_{b}$. Closed subanalytic sets that admit such a linear comparison of orders at every point form a class larger than Nash, which also satisfies the conditions of Theorem 3; a linear comparison at $b$ is equivalent to the property that the germ of $X$ at $b$ is the union of Nash and "formally embedded" non-Nash components (Theorem 7). Our proof of Theorem 3 shows that a uniform linear comparison (i.e., $\alpha_{b}, \beta_{b}$ bounded on compact subsets of $X$ ) is equivalent to the composite function property with linear loss of differentiability. (See $\S 4$.) We conjecture that these properties of $X$ follow from the conditions of Theorem 7 at each point.

\section{LOCAL PROPERTIES OF SUBANALYTIC SETS}

Notation. $\mathbb{N}$ denotes the nonnegative integers. Let $M$ be a real analytic manifold. Let $a \in M . \mathscr{O}_{M, a}$ or $\mathscr{O}_{a}$ denotes the ring of germs of analytic functions on $M$ at $a$, and $\hat{\mathscr{O}}_{a}$ the formal completion of $\mathscr{O}_{a}$. There is a natural homomorphism $f \mapsto \hat{f}_{a}$ from $\mathscr{C}^{\infty}(M)$ onto $\hat{\mathscr{O}}_{a}$, given by Taylor expansion in local coordinates. Suppose that $N$ is a real analytic manifold. Let $\varphi: M \rightarrow N$ be an analytic mapping. If $b=\varphi(a)$, there are local homomorphisms $\varphi_{a}^{*}: \mathscr{O}_{b} \rightarrow \mathscr{O}_{a}$ and $\hat{\varphi}_{a}^{*}: \hat{\mathscr{O}}_{b} \rightarrow \hat{\mathscr{O}}_{a}$ induced by composition with $\varphi$. In the following, $X$ denotes a closed subanalytic subset of $N$.

Lemma 1. Let $b \in X$. The following three definitions of $\mathscr{F}_{b}(X)$ are equivalent:

(1) Let $\varphi: M \rightarrow N$ be a proper analytic mapping such that $X=\varphi(M)$. Then $\mathscr{F}_{b}(X)=\bigcap_{a \in \varphi^{-1}(b)} \operatorname{Ker} \hat{\varphi}_{a}^{*}$.

(2) $\mathscr{F}_{b}(X)=\left\{G \in \hat{\mathscr{O}}_{b}:(G \circ \gamma)(t) \equiv 0\right.$ for every real analytic arc $\gamma(t)$ in $X$ such that $\gamma(0)=b\}$.

(3) $\mathscr{F}_{b}(X)=\left\{G \in \hat{\mathscr{O}}_{b}: T_{b}^{k} G(y)=o\left(|y-b|^{k}\right), y \in X\right.$, for all $k \in \mathbb{N}\}$. Here $T_{b}^{k} G(y)$ denotes the Taylor polynomial of order $k$ of $G$ in any local coordinate system.

We say that $X$ has the composite function property if, for any proper analytic mapping $\varphi: M \rightarrow N$ such that $X=\varphi(M)$, $\varphi^{*} \mathscr{C}^{\infty}(N)$ is closed in $\mathscr{C}^{\infty}(M)$; equivalently $\varphi^{*} \mathscr{C}^{\infty}(N)=$ $\varphi^{*} \mathscr{C}^{\infty}(N)^{\wedge}$, where $\varphi^{*} \mathscr{C}^{\infty}(N)^{\wedge}$ denotes the subalgebra of $\mathscr{C}^{\infty}(M)$ of functions $f$ such that, for all $b \in \varphi(M)$, there exists $G_{b} \in \hat{\mathscr{O}}_{b}$ with $\hat{f}_{a}=\hat{\varphi}_{a}^{*}\left(G_{b}\right)$ for every $a \in \varphi^{-1}(b)$. 
Formal semicoherence. We say that $X$ is formally semicoherent if it has a locally finite subanalytic stratification $X=\bigcup X_{i}$ (each stratum $X_{i}$ is understood to be smooth, connected, and locally closed) satisfying the following formal semicoherence property: Each point of $N$ admits a neighbourhood with coordinates $\left(y_{1}, \ldots, y_{n}\right)$ such that, for each $i$, there are finitely many formal power series $f_{i j}(\cdot, y)=\sum_{\alpha \in \mathbb{N}^{n}} f_{i j, \alpha}(\cdot) y_{1}^{\alpha_{1}} \cdots y_{n}^{\alpha_{n}}$ whose coefficients $f_{i j, \alpha}$ are analytic functions on $X_{i}$ which are subanalytic (i.e., their graphs are subanalytic as subsets of $N \times \mathbb{R}$ ) such that, for all $b \in X_{i}$ (in this neighbourhood), the $f_{i j}(b, y)$ generate the formal local ideal $\mathscr{F}_{b}(X)$. (Subanalyticity of the coefficients $f_{i j, \alpha}$ is a natural restriction on their growth at the boundary of the stratum.) Semicoherence was first studied by Galbiati [9] and Merrien [13] for real analytic or semianalytic sets.

The composite function and formal semicoherence properties of a subanalytic set are local, so we can assume $N=\mathbb{R}^{n}$. The coordinates of $\mathbb{R}^{n}$ will be denoted $\left(y_{1}, \ldots, y_{n}\right)$.

Chevalley-type estimates. Let $b \in X$ and $k \in \mathbb{N}$. Put

$$
\begin{array}{r}
\ell_{X}(b, k)=\min \left\{\ell \in \mathbb{N}: \text { if } f \in \mathscr{O}_{b} \text { and } \mu_{X, b}(f)>\ell,\right. \\
\text { then } \left.\nu_{X, b}(f)>k\right\} .
\end{array}
$$

Let $\varphi: M \rightarrow \mathbb{R}^{n}$ be a proper analytic mapping such that $X=$ $\varphi(M)$. Put

$$
\begin{array}{r}
\ell_{\varphi^{*}}(b, k)=\min \left\{\ell \in \mathbb{N}: \text { if } f \in \mathscr{O}_{b} \text { and } \nu_{M, a}\left(\varphi_{a}^{*}(f)\right)>\ell\right. \\
\text { for all } \left.a \in \varphi^{-1}(b) \text {, then } \nu_{X, b}(f)>k\right\} .
\end{array}
$$

If $\mathbf{a}=\left(a^{1}, \ldots, a^{s}\right)$ is an $s$-tuple of points in $\varphi^{-1}(b)$, put

$$
\begin{array}{r}
\ell_{\varphi_{a}^{*}}(k)=\min \left\{\ell \in \mathbb{N}: \quad \text { if } f \in \mathscr{O}_{b} \text { and } \nu_{M, a^{i}}\left(\varphi_{a^{i}}^{*}(f)\right)>\ell,\right. \\
\left.i=1, \ldots, s \text {, then } f \in \bigcap_{i=1}^{s} \operatorname{Ker} \hat{\varphi}_{a^{i}}^{*}+\hat{\mathfrak{m}}_{b}^{k+1}\right\} .
\end{array}
$$

(When $s=1$, a is a point $a \in M$, and $\ell_{\varphi_{a}^{*}}(k)$ depends only on $\varphi_{a}^{*}$.) Then $\ell_{\varphi_{\mathrm{a}}^{*}}(k)<\infty$, by a classical lemma of Chevalley $[4,8.2 .2]$. If a includes a point in every connected component of $\varphi^{-1}(b)$, then $\bigcap_{i=1}^{s} \operatorname{Ker} \hat{\varphi}_{a^{i}}^{*}=\mathscr{F}_{b}(X)[4,11.1]$ and $\ell_{\varphi^{*}}(b, k) \leq$ $\ell_{\varphi_{\mathrm{a}}^{*}}(k)$. 
Lemma 2. Let $\varphi: M \rightarrow \mathbb{R}^{n}$ be a proper analytic mapping such that $X=\varphi(M)$.

(1) $\ell_{X}(b, \cdot) \leq \ell_{\varphi^{*}}(b, \cdot)$, for all $b \in X$.

(2) If $K \subset X$ is compact, then $\ell_{\varphi^{*}}(b, \cdot) \leq r \ell_{X}(b, \cdot)$, for all $b \in K$, where $r \geq 1$.

The diagram of initial exponents and the Hilbert-Samuel function. Let $b \in \mathbb{R}^{n}$. Identify $\hat{\mathscr{O}}_{b}$ with $\mathbb{R} \llbracket y-b \rrbracket$. If $\alpha=\left(\alpha_{1}, \ldots, \alpha_{n}\right) \in$ $\mathbb{N}^{n}$, put $|\alpha|=\alpha_{1}+\cdots+\alpha_{n}$. The lexicographic ordering of $(n+$ 1)-tuples $\left(|\alpha|, \alpha_{1}, \ldots, \alpha_{n}\right)$ induces a total ordering of $\mathbb{N}^{n}$. Let $f \in \mathbb{R} \llbracket y-b \rrbracket$. Write $f=\sum_{\alpha \in \mathbb{N}^{n}} f_{\alpha}(y-b)^{\alpha}$, where $(y-b)^{\alpha}$ denotes $\left(y_{1}-b_{1}\right)^{\alpha_{1}} \cdots\left(y_{n}-b_{n}\right)^{\alpha_{n}}$. The initial exponent $\exp f$ means $\min \left\{\alpha \in \mathbb{N}^{n}: f_{\alpha} \neq 0\right\}$.

Let $I$ be an ideal in $\mathbb{R} \llbracket y-b \rrbracket$. The diagram of initial exponents $\mathfrak{N}(I) \subset \mathbb{N}^{n}$ is defined as $\mathfrak{N}(I)=\{\exp f: f \in I\}$. Put $\mathscr{D}(n)=$ $\left\{\mathfrak{N} \subset \mathbb{N}^{n}: \mathfrak{N}+\mathbb{N}^{n}=\mathfrak{N}\right\}$. Clearly, $\mathfrak{N}(I) \in \mathscr{D}(n)$. We totally order $\mathscr{D}(n)$ as follows: To each $\mathfrak{N} \in \mathscr{D}(n)$, associate the sequence $v(\mathfrak{N})$ obtained by listing the "vertices" $\{\alpha \in \mathfrak{N}: \mathfrak{N}-\{\alpha\} \in \mathscr{D}(n)\}$ in ascending order and completing the list to an infinite sequence by using $\infty$ for all the remaining terms. If $\mathfrak{N}^{1}, \mathfrak{N}^{2} \in \mathscr{D}(n)$, we say that $\mathfrak{N}^{1}<\mathfrak{N}^{2}$ provided that $v\left(\mathfrak{N}^{1}\right)<v\left(\mathfrak{N}^{2}\right)$ with respect to the lexicographic ordering on the set of all such sequences.

If $I$ is an ideal in $\hat{\mathscr{O}}_{b}=\mathbb{R} \llbracket y-b \rrbracket$, then the Hilbert-Samuel function $H: \mathbb{N} \rightarrow \mathbb{N}$ of $\hat{\mathscr{O}}_{b} / I$ is defined as $H(k)=\operatorname{dim}_{\mathbb{R}} \hat{\mathscr{O}}_{b} /(I+$ $\left.\hat{\mathfrak{m}}_{b}^{k+1}\right)$. It follows from Hironaka's formal division algorithm [4, $\S 6]$ that $H(k)$ is the number of elements $\alpha \in \mathbb{N}^{n}$ such that $\alpha \notin$ $\mathfrak{N}(I)$ and $|\alpha| \leq k$. The set $\mathbb{N}^{\mathbb{N}}$ of functions from $\mathbb{N}$ to itself is partially ordered as follows: If $H, H^{\prime} \in \mathbb{N}^{\mathbb{N}}$, then $H<H^{\prime}$ if $H(k) \leq H^{\prime}(k)$ for all $k$ and $H(k)<H^{\prime}(k)$ for some $k$.

Subanalytic semicontinuity. Let $X \supset Z$ be closed subanalytic subsets of $\mathbb{R}^{n}$, and let $\Sigma$ be a partially ordered set. We say that a function $\tau: X \rightarrow \Sigma$ is subanalytic Zariski (upper-) semicontinuous rel. $Z$ if: (1) For every compact $K \subset X$, there are only finitely many values of $\tau(b), b \in(X-Z) \cap K$. (2) For all $\sigma \in \Sigma$, $Z_{\sigma}=Z \cup\{b \in X-Z: \tau(b) \geq \sigma\}$ is a closed subanalytic set.

The main theorem. If $L$ is a closed subset of a real analytic manifold $M$, let $\mathscr{C}^{\infty}(M ; L)$ denote the closed subalgebra of $\mathscr{C}^{\infty}(M)$ consisting of functions that are flat on $L$. Suppose that $\varphi: M \rightarrow$ $\mathbb{R}^{n}$ is a proper analytic mapping and that $Z$ is a closed 
subset of $\mathbb{R}^{n}$. Then $\varphi^{*}: \mathscr{C}^{\infty}\left(\mathbb{R}^{n}\right) \rightarrow \mathscr{C}^{\infty}(M)$ induces $\varphi^{*}$ : $\mathscr{C}^{\infty}\left(\mathbb{R}^{n} ; Z\right) \rightarrow \mathscr{C}^{\infty}\left(M ; \varphi^{-1}(Z)\right)$.

Theorem 3. Let $X \subset Z$ be closed subanalytic subsets of $\mathbb{R}^{n}$. Then the following properties of the pair $(X, Z)$ are equivalent:

(1) Composite function property. If $M$ is a real analytic manifold and $\varphi: M \rightarrow \mathbb{R}^{n}$ is a proper analytic mapping such that $X=\varphi(M)$, then $\varphi^{*} \mathscr{C}^{\infty}\left(\mathbb{R}^{n} ; Z\right)=\varphi^{*} \mathscr{C}^{\infty}\left(\mathbb{R}^{n} ; Z\right)^{\wedge}$ (where $\varphi^{*} C^{\infty}\left(\mathbb{R}^{n} ; Z\right)^{\wedge}$ means $\left.\varphi^{*} \mathscr{C}^{\infty}\left(\mathbb{R}^{n}\right)^{\wedge} \cap \mathscr{C}^{\infty}\left(M ; \varphi^{-1}(Z)\right)\right)$.

(2) Uniform Chevalley estimate. For every compact subset $K$ of $\mathbb{R}^{n}$, there is a function $\ell_{K}: \mathbb{N} \rightarrow \mathbb{N}$ such that $\ell_{X}(b, k) \leq \ell_{K}(k)$, for all $b \in(X-Z) \cap K$ and $k \in \mathbb{N}$.

(3) Semicontinuity of the diagram of initial exponents. Let $\mathfrak{N}_{b}=$ $\mathfrak{N}\left(\mathscr{F}_{b}(X)\right), b \in X$. Then $\mathfrak{N}_{b}$, as a function $X \rightarrow \mathscr{D}(n)$, is subanalytic Zariski semicontinuous rel. $Z$.

(4) Semicontinuity of he Hilbert-Samuel function. Let $H_{b}$ be the Hilbert-Samuel function of $\hat{\mathscr{O}}_{b} / \mathscr{F}_{b}(X), b \in X$. Then the function $b \mapsto H_{b}$ from $X$ to $\mathbb{N}^{\mathbb{N}}$ is subanalytic Zariski semicontinuous rel. $Z$.

(5) Formal semicoherence. $X$ admits a locally finite subanalytic stratification $X=\bigcup X_{i}$ such that $Z$ is a union of strata and $X$ satisfies the formal semicoherence property along every stratum outside $Z$.

The extension property. Let $Z$ be a closed subset of $X$. Let $\mathscr{C}^{\infty}(X ; Z)$ denote $\mathscr{C}^{\infty}\left(\mathbb{R}^{n} ; Z\right) /\left\{f \in \mathscr{C}^{\infty}\left(\mathbb{R}^{n} ; Z\right): f=0\right.$ on $X\}$. Of course, if $\varphi: M \rightarrow \mathbb{R}^{n}$ is a proper analytic mapping such that $X=\varphi(M)$, then $\left\{f \in \mathscr{C}^{\infty}\left(\mathbb{R}^{n}\right): f=0\right.$ on $\left.X\right\}=\operatorname{Ker} \varphi^{*}$. An extension operator $E: \mathscr{C}^{\infty}(X ; Z) \rightarrow \mathscr{C}^{\infty}\left(\mathbb{R}^{n} ; Z\right)$ is, by definition, a continuous linear splitting of the canonical surjection $\mathscr{C}^{\infty}\left(\mathbb{R}^{n} ; Z\right) \rightarrow \mathscr{C}^{\infty}(X ; Z)$.

Corollary 4. Let $X \supset Z$ be closed subanalytic subsets of $\mathbb{R}^{n}$. If $(X, Z)$ satisfies the conditions of Theorem 3 , then there is an extension operator $E: \mathscr{C}^{\infty}(X ; Z) \rightarrow \mathscr{C}^{\infty}\left(\mathbb{R}^{n} ; Z\right)$.

Corollary 5. If $X$ (i.e., $(X, \varnothing))$ satisfies the conditions of Theorem 3 and $b \in X$, then $\mathscr{F}_{b}(X)$ is the ideal of formal Taylor expansions of $\mathscr{C}^{\infty}$ functions that vanish on $X$.

From an analytic viewpoint, Theorem 3 concerns the solution of an equation of the form $f(x)=g(\varphi(x))$, where $\varphi$ is a given analytic mapping and $f$ a given $\mathscr{C}^{\infty}$ function. There is a more 
general "module" version of Theorem 3 that applies to systems of equations of the form $f(x)=A(x) \cdot g(\varphi(x))+B(x) \cdot h(x)$, where $A(x), B(x)$ are given matrices of analytic functions, $f(x)$ is a given (vector-valued) $\mathscr{C}^{\infty}$ function, and $g(y), h(x)$ are the unknowns (cf. [4]).

\section{IMPORTANT CLASSES OF SUBANALYTIC SETS}

Invariants of Gabrielov. Theorem 3 provides an interesting parallel to the following theorem, due essentially to Gabrielov, concerning the solution of equations of the form $f(x)=g(\varphi(x))$, where $\varphi$ and $f$ are given analytic germs. (See $[1,8,12,14]$.) Let $\mathbb{K}=\mathbb{R}$ or $\mathbb{C}$. Let $\varphi$ be an analytic mapping from a neighbourhood of 0 in $\mathbb{K}^{m}$ to $\mathbb{K}^{n}$, such that $\varphi(0)=0$. Let $r_{0}^{1}(\varphi)$ be the generic rank of $\varphi$ near 0 . Put $r_{0}^{2}(\varphi)=\operatorname{dim} \hat{\mathscr{O}}_{\mathbb{K}^{n}, 0} / \operatorname{Ker} \hat{\varphi}_{0}^{*}$, $r_{0}^{3}(\varphi)=\operatorname{dim} \mathscr{O}_{\mathbb{K}^{n}, 0} / \operatorname{Ker} \varphi_{0}^{*}$ (dim denotes the Krull dimension). It is easy to see that $r_{0}^{1}(\varphi) \leq r_{0}^{2}(\varphi) \leq r_{0}^{3}(\varphi)$.

Theorem 6. The following conditions are equivalent:

(1) Compositefunction property. $\hat{\varphi}_{0}^{*}\left(\hat{\mathscr{O}}_{\mathbb{K}^{n}, 0}\right) \cap \mathscr{O}_{\mathbb{K}^{m}, 0}=\varphi_{0}^{*}\left(\mathscr{O}_{\mathbb{K}^{n}, 0}\right)$.

(2) Linear Chevalley estimate. There exist $\alpha \geq 1, \beta \geq 0$ such that $\ell_{\varphi_{0}^{*}}(k) \leq \alpha k+\beta$, for all $k \in \mathbb{N}$.

(3) $r_{0}^{1}(\varphi)=r_{0}^{2}(\varphi)$; i.e., there are "sufficiently many" formal relations.

(4) $r_{0}^{1}(\varphi)=r_{0}^{3}(\varphi)$. (We say that $\varphi$ is regular at 0 .)

A subanalytic set is called Nash if it is a locally finite union of pure-dimensional subanalytic sets each of which lies in an analytic set of the same dimension. Thus, closed Nash subanalytic sets are precisely the images of proper real analytic mappings which are regular at every point.

Theorem 7. Let $X$ be a closed subanalytic subset of $\mathbb{R}^{n}$. Let $b \in$ $X$. Then the following conditions are equivalent:

(1) There exist $\alpha \geq 1$ and $\beta \geq 0$ such that $\ell_{X}(b, k) \leq \alpha k+\beta$, for all $k$.

(2) $\mathscr{F}_{b}(X)=\mathscr{F}_{b}(Y)$, where $Y$ is some closed Nash subanalytic subset of $X$.

Moreover, if $Z$ is a closed subanalytic subset of $X$, and $X$ satisfies the conditions above at each point of $X-Z$, then $(X, Z)$ satisfies the properties of Theorem 3.

Suppose that $\varphi: M \rightarrow \mathbb{R}^{n}$ is a proper real analytic mapping. In [4] we proved that uniformity of the Chevalley estimate 
$\ell_{\varphi_{a}^{*}}(k)$ is equivalent to analytic Zariski-semicontinuity (on $M$ ) of the diagram $\mathfrak{N}\left(\operatorname{Ker} \hat{\varphi}_{a}^{*}\right)$ (or of the Hilbert-Samuel function of $\left.\hat{\mathscr{O}}_{\varphi(a)} / \operatorname{Ker} \hat{\varphi}_{a}^{*}\right)$, and, moreover, that these conditions imply that

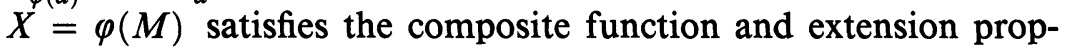
erties. These conditions are satisfied when $\varphi$ is regular at every point $[4, \S 13]$. We can construct a closed subanalytic set $X$ satisfying the conditions of Theorem 7 at every point, which is the image of no proper real analytic mapping admitting a uniform Chevalley estimate $\ell_{\varphi_{a}^{*}}(k)$. For example, $X=P \cup H$, where $P$ is Pawłucki's example [15] and $H$ is a closed half-space whose boundary contains the line of non-Nash points in $P$.

\section{REMARKS ON THE PROOF}

While the implication $(1) \Rightarrow(2)$ in Theorem 3 is striking, it has a simple proof: The topology of $\mathscr{C}^{\infty}(M)$ is defined by a system of seminorms $\|f\|_{L}^{\ell}$ giving the supremum of the derivatives of order up to $\ell$ of $f$ on compact subsets $L$ of $M$. The seminorms $\|g\|_{K}^{k}$ for $\mathscr{C}^{\infty}\left(\mathbb{R}^{n}\right)$ induce seminorms $\|[g]\|_{K}^{k}=\min \left\{\|g+h\|_{K}^{k}: h \in\right.$ $\left.\operatorname{Ker} \varphi^{*}\right\}$ on $\mathscr{C}^{\infty}\left(\mathbb{R}^{n}\right) / \operatorname{Ker} \varphi^{*}$. Assume (1). (For simplicity, suppose $Z=\varnothing$.) By the open mapping theorem, for every compact $K \subset X$ and every $k \in \mathbb{N}$, there exists $\ell=\ell(K, k) \in \mathbb{N}$ such that $\|[g]\|_{K}^{k} \leq$ const $\left\|\varphi^{*}(g)\right\|_{\varphi^{-1}(K)}^{\ell}, g \in \mathscr{C}^{\infty}\left(\mathbb{R}^{n}\right)$. Then $\ell_{\varphi^{*}}(b, k) \leq \ell(K, k)$, for all $b \in K$ : Suppose this is false. Then, for some $b \in K$, there exist $G \in \hat{\mathscr{O}}_{b}$ and $k \in \mathbb{N}$ such that $\hat{\varphi}_{a}^{*}(G) \in \hat{m}_{a}^{\ell+1}$, for all $a \in \varphi^{-1}(b)$, where $\ell=\ell(K, k)$, but $G \notin \mathscr{F}_{b}(X)+\hat{\mathfrak{m}}_{b}^{k+1}$. Let $g \in \mathscr{C}^{\infty}\left(\mathbb{R}^{n}\right)$ such that $\hat{g}_{b}=G$. We can assume that $\|[g]\|_{\{b\}}^{k}=1$. For any $\epsilon>0$, there is a function $\alpha_{\epsilon} \in \mathscr{C}^{\infty}\left(\mathbb{R}^{n}\right)$ such that (i) $0 \leq \alpha_{\epsilon} \leq 1, \alpha_{\epsilon}(y)=1$ near $b$, and $\alpha_{\epsilon}(y)=0$ outside $\{|y-b|<\epsilon\}$; (ii) the derivatives of order $k$ of $\alpha_{\epsilon}$ are bounded uniformly by constant $/ \epsilon^{k}, k \in \mathbb{N}$. Then $\left\|\left[\alpha_{\epsilon} g\right]\right\|_{K}^{k} \geq 1$ but $\lim _{\epsilon \rightarrow 0}\left\|\varphi^{*}\left(\alpha_{\epsilon} g\right)\right\|_{\varphi^{-1}(K)}^{\ell}=0$; a contradiction.

In particular, if the composition property holds with linear loss of differentiability, i.e., $\ell(K, k) \leq \alpha_{K} k+\beta_{K}$, for every compact $K \subset X$, then there is a uniform linear Chevalley estimate $\ell_{X}(b, k) \leq \alpha_{K} k+\beta_{K}, b \in K$. The converse also follows from the techniques we use to prove Theorem 3. 


\section{REFERENCES}

1. J. Becker and W. R. Zame, Applications of functional analysis to the solution of power series equations, Math. Ann. 243 (1979), 37-54.

2. E. Bierstone and P. D. Milman, Composite differentiable functions, Ann. of Math. 116 (1982), 541-558.

3. E. Bierstone and P. D. Milman, The Newton diagram of an analytic morphism, and applications to differentiable functions, Bull. Amer. Math. Soc. (N.S.) 9 (1983), 315-318.

4. E. Bierstone and P. D. Milman, Relations among analytic functions I, II, Ann. Inst. Fourier (Grenoble) 37 (1) (1987), 187-239; and 37 (2) (1987), 49-77.

5. E. Bierstone and P. D. Milman, Local analytic invariants and splitting theorems in differential analysis, Israel J. Math. 60 (1987), 257-280.

6. E. Bierstone and P. D. Milman, Semianalytic and subanalytic sets, Inst. Hautes Etudes Sci. Publ. Math. 67 (1988), 5-42.

7. A. M. Gabrielov, Formal relations between analytic functions, Functional Anal. Appl. 5 (1971), 318-319.

8. A. M. Gabrielov, Formal relations between analytic functions, USSR Izv. 7 (1973), 1056-1088.

9. M. Galbiati, Stratifications et ensemble de non-cohérence d'un espace analytique réel, Invent. Math. 34 (1976), 113-128.

10. G. Glaeser, Fonctions composées différentiables, Ann. of Math. 77 (1963), 193-209.

11. H. Hironaka, Local analytic dimensions of a subanalytic set, Proc. Japan Acad. Ser. A Math. Sci. 62 (1986), 73-75.

12. S. Izumi, Gabrielov's rank condition is equivalent to an inequality of reduced orders, Math. Ann. 276 (1986), 81-89.

13. J. Merrien, Faisceaux analytiques semi-cohérents, Ann. Inst. Fourier (Grenoble) 30 (1980), 165-219.

14. P. D. Milman, Analytic and polynomial homomorphisms of analytic rings, Math. Ann. 232 (1978), 247-253.

15. W. Pawłucki, On relations among analytic functions and geometry of subanalytic sets, Bull. Polish Acad. Sci. Math. 37 (1989), 117-125.

Department of Mathematics, University of Toronto, Toronto, Ontario, Canada M5S 1A1 
